\title{
Coexistence of Orthogonal and Non-orthogonal Multicarrier Signals in Beyond 5G Scenarios
}

\author{
Xinyue Liu, Tongyang Xu, and Izzat Darwazeh \\ Department of Electronic and Electrical Engineering \\ University College London \\ London, United Kingdom \\ Email: x.liu.17@ucl.ac.uk, tongyang.xu.11@ucl.ac.uk, i.darwazeh@ucl.ac.uk
}

\begin{abstract}
Optimum operation of future mobile communication systems requires more flexible signalling mechanisms for radio access. For flexible heterogeneous signalling implementation, this work discusses coexistence scenarios of orthogonal and nonorthogonal multicarrier signals, specifically considering orthogonal frequency division multiplexing (OFDM) and spectrally efficient FDM (SEFDM) signals. Three main scenarios of the coexisting signalling are addressed under $5 \mathrm{G}$ new radio (5G NR) numerology with varying subcarrier spacing. Using numerical simulations, this work reports performance results of systems operating under the studied coexistence scenarios assuming uncoded and coded signals. Results reveal that systems employing SEFDM and OFDM result in some BER degradation when uncoded signals are used and also show that when applying low-density parity-check (LDPC) to the transmitted signals, the coexistence effects are mitigated and the block error rate (BLER) for both orthogonal and non-orthogonal signals suffers only slight degradation.
\end{abstract}

Index Terms-Coexistence scenario, 5G NR numerology, SEFDM, non-orthogonal waveform, OFDM, LDPC

\section{INTRODUCTION}

To meet the requirements of the use scenarios in IMT-2020, the $3^{\text {rd }}$ generation partnership project (3GPP) proposed the new radio-access standard for $5 \mathrm{G}$ system, termed as $5 \mathrm{G}$ new radio (5G NR) [1] [2]. One of the primary purposes of 5G NR is to support heterogeneous system implementation. Hence, higher flexibility is needed so that different services can be provided to devices using the same radio resources. As such, the feasibility of coexistence of different signalling formats, configured in the same range of time-frequency resources, needs to be studied and assessed.

5G NR maintains the orthogonal frequency division multiplexing (OFDM) waveform format (as used in LTE [3]) as specified in release 15 [1]. To handle different scenarios, 5G NR offers higher spectrum flexibility through defining flexible numerology for its physical layer specifications. Rather than the fixed subcarrier spacing (SCS) of LTE, 5G NR supports five sets of SCSs of 15/30/60/120/240 kHz.

Although OFDM is the dominant signalling format that serves $5 \mathrm{G} \mathrm{NR}$, some other methods are being considered for beyond $5 \mathrm{G}$ scenarios. Over the past decade several waveforms/signalling technologies have been studied and tested, to serve the high-data-rate low-latency communications. These include filter bank multi-carrier (FBMC) [4], generalized frequency division multiplexing (GFDM) [5] and universal filtered multi-carrier (UFMC) [6] proposed to improve performance, enhance spectral efficiency and/or suppress out-ofband $(\mathrm{OOB})$ emission.

Over the past two decades, other non-orthogonal signalling techniques, with significant increase in spectral efficiency, have been investigated by academia and industry [7]. A prominent time-domain technique, termed as multi-stream faster-thanNyquist signalling (FTN), gains up to $25 \%$ data rate improvement without error performance degradation [8]. Sharing the same goal, spectrally efficient flexible waveform termed as spectrally efficient FDM (SEFDM), firstly proposed in 2003 [9], has been demonstrated to offer up to $40 \%$ spectral efficiency improvement [10] [11] with little performance degradation. For specific use case such as narrowband Internet of Things (NB-IoT), the semi-orthogonal Fast-OFDM technique, a variant of SEFDM, offers $100 \%$ spectral efficiency gains using one dimensional modulations [12]. For specially designed waveform, quadrupling the bit rate within the same bandwidth has been demonstrated recently, when used with the one dimensional modulation scheme BPSK [13].

To envision the forthcoming $5 \mathrm{G}$ NR design that allows improvement on both spectral efficiency and system flexibility, we explore three coexistence scenarios, involving simultaneous transmission of different signals in adjacent frequency bands (i.e. adjacent bandwidth parts-BWP) and without guard bands, therefore some overlap at the band edges is assumed to result in worst case scenario studies. Signals used are orthogonal and non-orthogonal waveforms of different SCSs and spectral efficiencies. The work starts with studying the bit error rate (BER) of raw data without coding. Then standard compliant LDPC coding is applied to the OFDM 5G NR system and with minor modifications to the non-orthogonal signals. In this case, coexistence effects are presented using the block error rate (BLER). The signal reception and detection is implemented assuming independent data transmission and ideal channel effects.

This paper is organised as follows: section II introduces nonorthogonal SEFDM signals and outlines their key advantages. This is followed by a brief description of three main coexistence scenarios of OFDM and SEFDM waveforms. Section III reports the modelling results and performance of systems with uncoded and LDPC coded coexisting signals. Section IV concludes the paper. 


\section{SySTEM MOdEL UNDER 5G NR NUMEROLOGY}

\section{A. Non-orthogonal SEFDM Waveform}

SEFDM is a non-orthogonal multicarrier waveform, achieving better bandwidth utilisation by reducing the spacing between neighbouring subcarriers. It promises higher spectral efficiency as compared to OFDM system at the expense of added inter-carrier interference (ICI), which is one of the major challenges in the development of SEFDM system.

The initial idea of SEFDM signal generation is based on the compression of the frequency separation between subcarriers. Similar to OFDM, SEFDM can be generated using inverse fast Fourier transform (IFFT) with appropriate modifications [14]. Relative to OFDM, SEFDM compresses the bandwidth by factor $\alpha$ and thus improves the spectral efficiency by $(1 / \alpha-1) \times 100 \%$ [9]. Fig. 1 shows the subcarrier representation of the SEFDM signal in the format of type-I SEFDM [15]. Another form of SEFDM termed as type-II SEFDM [15] achieves higher spectral efficiency by increasing the data rate for each subcarrier while keeping the subcarrier separation the same as OFDM. In this work, to ensure that same transmission rate is achieved, type-I SEFDM signal is employed.

Since the orthogonality is deliberately violated and consequently the self-introduced ICI compromises the signal recovery, advanced detection algorithms are required to maintain good BER performance. Sphere decoder (SD) was first applied to SEFDM showing significant improvement in error performance within the range of poor signal-to-noise ratio (SNR) [16]. Other detection techniques such as fixed SD (FSD) [14] and generalised SD (GSD) [16] were employed to reduce receiver complexity. A brief survey on SEFDM is provided in [7], covering the techniques that have been applied to SEFDM including signal generation, reception, channel estimation and equalisation. The main advantages of SEFDM system are listed below:

1) High Spectral Efficiency: The most appealing advantage of SEFDM concept over OFDM is the enhanced bandwidth efficiency. A clear bandwidth reduction can be seen in Fig. 1 for the SEFDM signal when compared to the OFDM signal. Information is transmitted at same data rate. Furthermore, the
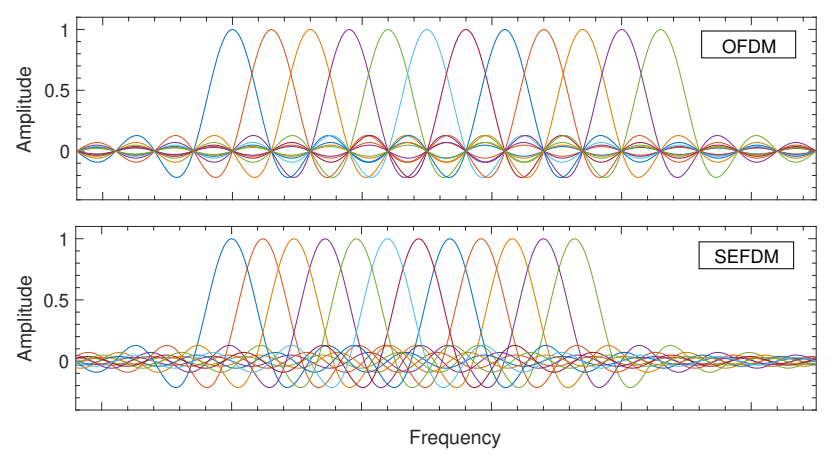

Fig. 1. OFDM (top) and type-I SEFDM (bottom) subcarrier representation (N $=12$ ), wherein the bandwidth compression factor $\alpha$, the ratio of the subcarrier spacing $\triangle f_{S E F D M} / \Delta f_{O F D M}$, is set to 0.8 .

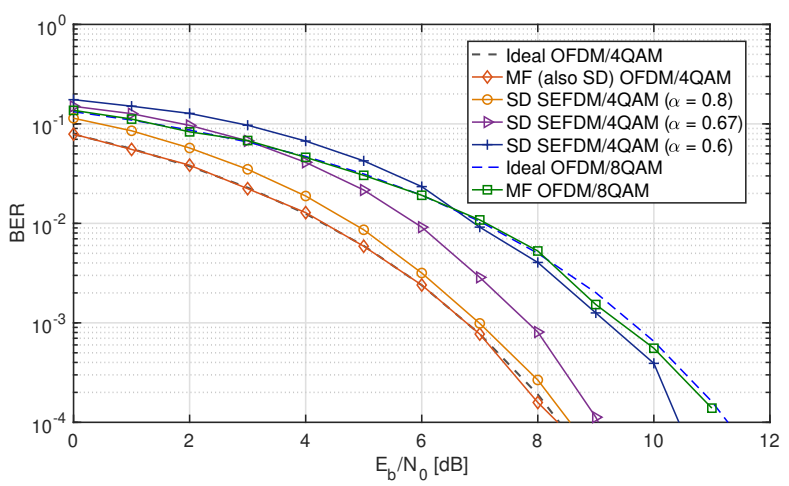

Fig. 2. BER performance for uncoded SEFDM and OFDM systems.

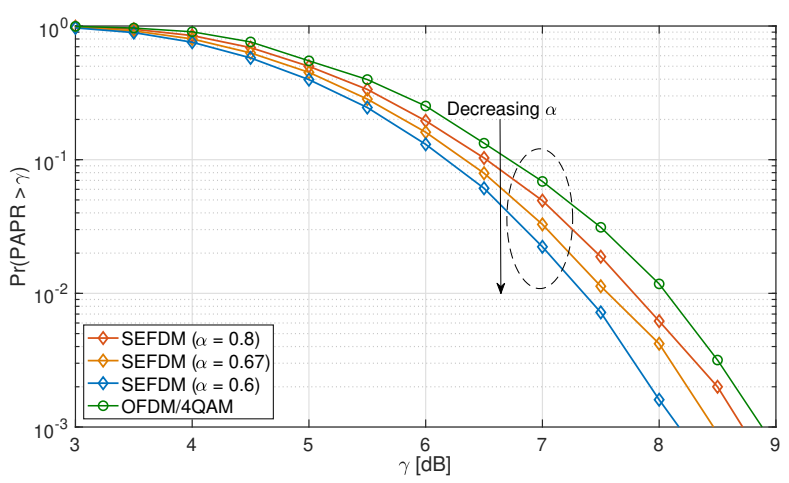

Fig. 3. Comparison of CCDF of PAPR for OFDM and SEFDM with varying values of $\alpha$.

spectral efficiency can be doubled by applying Hilbert filter pair to SEFDM [17].

2) Maintained BER: Many studies of BER performance of SEFDM with different detection techniques have been performed [7]. Fig. 2 provides a good summary in simulations showing the BER for SEFDM system under 4QAM modulation with varying values of $\alpha=0.8,0.67$ and 0.6. The SEFDM signal is detected using the SD algorithm. It can be seen in Fig. 2 that the SEFDM with $\alpha=0.6$ has similar BER as the OFDM modulated with 8QAM. Besides, the SEFDM with $\alpha=$ 0.8 obtains approximately the same BER as OFDM modulated with $4 \mathrm{QAM}$ when the $E_{b} / N_{0}$ is higher than $5 \mathrm{~dB}$.

3) Low PAPR: Another benefit of SEFDM, when compared to OFDM system, is the reduced peak-to-average-power ratio (PAPR). In [18] detailed investigations have been provided for the PAPR of SEFDM signal and the effects of PAPR reduction techniques associated with OFDM on SEFDM system. We have reproduced the results, shown in Fig. 3. The complementary cumulative distribution function (CCDF) of PAPR of OFDM signal modulated with 4 QAM is given for comparison. This figure depicts the CCDF of the PAPR of the SEFDM/4QAM signal exceeding a threshold of $\gamma$ for different value of $\alpha$. The probability increases expectedly with the reduction of $\alpha$, which indicates the improving power efficiency. 
TABLE I

THREE TYPES OF COEXISTENCE SCENARIO UNDER 5G NR NUMEROLOGIES

\begin{tabular}{llllllll}
\hline Scenario & Signal 1 & $\alpha_{1}$ & $\begin{array}{c}\Delta f_{1} \\
{[\mathrm{kHz}]}\end{array}$ & Signal 2 & $\alpha_{2}$ & $\begin{array}{l}\Delta f_{2} \\
{[\mathrm{kHz}]}\end{array}$ \\
\hline Senario-I & OFDM & 1 & 15 & OFDM & 1 & $\begin{array}{l}15 / 30 / 60 \\
/ 120 / 240\end{array}$ \\
\hline \multirow{2}{*}{ Senario-II } & OFDM & \multirow{2}{*}{1} & \multirow{2}{*}{15} & & SEFDM & 0.67 & 10 \\
\cline { 5 - 7 } & & & & & SEFDM & 0.6 & 9 \\
\cline { 5 - 7 } Senario-III & SEFDM & 0.8 & 15 & SEFDDM & 0.8 & 12 \\
\cline { 4 - 7 } & & & & SEFDM & 0.6 & 9 \\
\hline
\end{tabular}

These advantages of SEFDM, coupled with new efficacious techniques for channel estimation and equalisation [19] [20] [21], make SEFDM an attractive candidate for future communication systems derived from and coexisting with 5G NR.

\section{B. Discussion on Coexistence Scenarios}

The ultimate goal of this work is to investigate the effects of coexisting signals when they are transmitted simultaneously. To this purpose, we categorise coexisting signalling into three main scenarios given by Table I: OFDM-OFDM, OFDM-SEFDM and SEFDM-SEFDM coexistence. In order to simplify the problem, we assume that two signals - signal 1 with SCS of $\Delta f_{1}$ and signal 2 with SCS of $\Delta f_{2}$ are transmitted and received by different UE. The two signals are independent; however, they are allocated BWP without guard band. It is worth noting in Table I that the $\alpha$ for SEFDM signal is noted to show the reduced SCS when compared to OFDM, of which the underlying $\alpha=1$.

In more detailed terms, in scenario-I we define that the two OFDM signals are of different SCS. In order to assess the effects of applying flexible SCS, we maintain one of the OFDM signal with fixed SCS $\Delta f_{1}=15 \mathrm{kHz}$ meanwhile vary the coexisting signal SCS $\Delta f_{2}=15,30,60,120,240 \mathrm{kHz}$. Scenario-II is more complicated since different waveforms are considered, where OFDM and SEFDM signals coexist occupying the neighbouring BWPs. Scenario-III specifies the coexistence of two non-orthogonal signals; one has fixed $\alpha_{1}=0.8$ and the other's $\alpha_{2}$ varies. To illustrate, we use scenario-II as an example in Fig. 4.

\section{Scenario-based Performance Assessment}

This section presents the numerical simulation results commencing with the specifications of the system models. The BER performance for signals transmitted in three different scenarios is evaluated for the uncoded systems. Besides, we assess the BLER against practical SNR for the same systems with LDPC coding.

\section{A. System Configurations}

For simplicity, we employ 12 subcarriers modulated with 4QAM scheme for both the OFDM and SEFDM signal. This

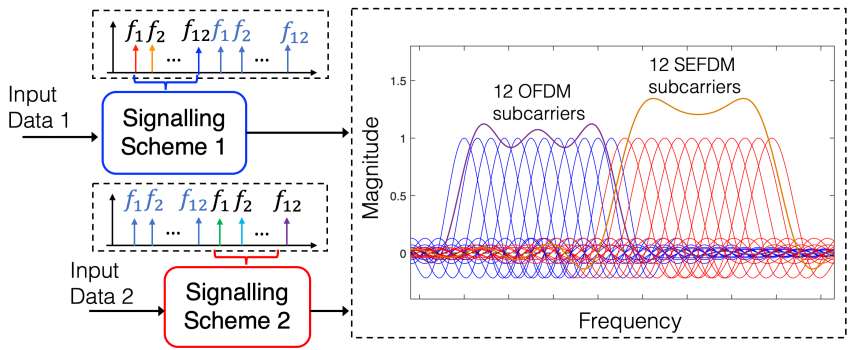

Fig. 4. Coexisting Scenario-II OFDM-SEFDM subcarrier representation. For Scenario-I and Scenario-III, similar subcarrier placement with different parameters will be used.

follows the standard where each resource block consists of 12 subcarriers [1]. The centre carrier frequency is set to 3.5 $\mathrm{GHz}$ which is in the new spectrum range included in the $5 \mathrm{G}$ NR technology specifications. This is to match the $5 \mathrm{G}$ numerologies that are used for the flexible SCS settings.

\section{B. BER Performance Evaluation}

The BER performance for the single-antenna systems in the three different scenarios is examined for different values of $E_{b} / N_{0}$. Extensions to multi-antenna is straightforward with minor system architecture modifications [15]. For comparison, the ideal error performance for OFDM modulated with 4QAM scheme is provided in Figs. 6-8. The simulations assume that the received signal is contaminated only by additive white Gaussian noise (AWGN).

For scenario-I, Fig. 5 displays the power spectra for the two independent OFDM signals with normalised amplitude and frequency. Numerical simulations are carried out to assess the performance of coexisting OFDM signals with different values of SCS as listed in Table I. The signal detection at the receiver employs matched filtering and the corresponding BER curves of the associated OFDM signals are presented in Fig. 6. Apart from the first coexisting system where $\Delta f_{1}=\Delta f_{2}=15 \mathrm{kHz}$, evident BER degradation appears in the rest of coexisting systems due to the inter-numerology interference. The signals with large SCS are robust to the inter-numerology interference while those with small subcarrier spacing are more susceptible to the interference resulting in error floors when $E_{b} / N_{0}$ is further increased.

TABLE II

SPECIFICATIONS FOR THE NUMERICAL SIMULATED COEXISTENCE SYSTEM

\begin{tabular}{lll}
\hline Parameter & OFDM & SEFDM \\
\hline \hline Centre Carrier frequency $[\mathrm{GHz}]$ & 3.5 & 3.5 \\
\hline Sampling Frequency $[\mathrm{MHz}]$ & 1.92 & 1.92 \\
\hline IFFT Output Size & 128 & 128 \\
\hline Number of Subcarriers & 12 & 12 \\
\hline Modulation Scheme & 4QAM & 4QAM \\
\hline Bandwidth Compression Factor $\alpha$ & 1 & $0.8,0.67,0.6$ \\
\hline Data Bandwidth [kHz] & 180 & $144,120,108$ \\
\hline Data Rate [kbit/s] & 180 & 180 \\
\hline \hline
\end{tabular}




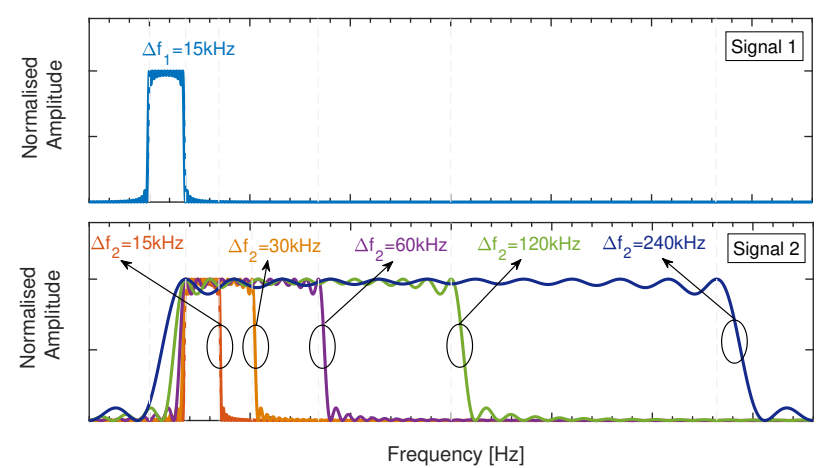

Fig. 5. Scenario-I: power spectrum with normalised amplitude for OFDM signal 1 (top) and OFDM signal 2 with varying SCS (bottom).

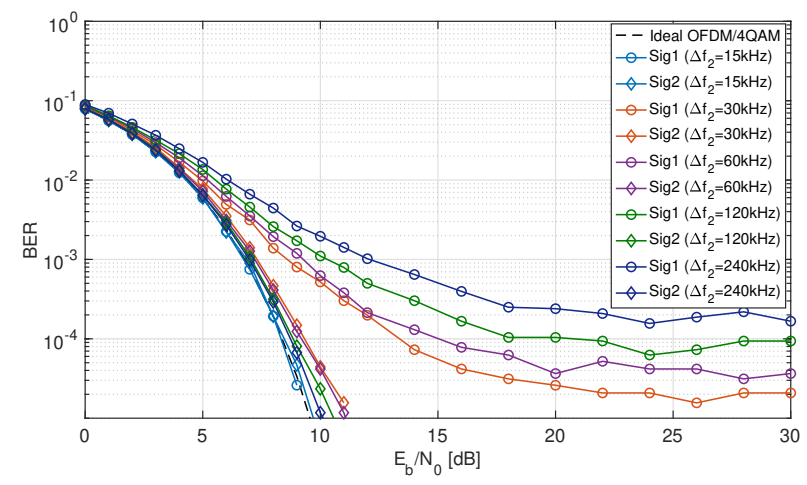

Fig. 6. Scenario-I: BER performance for OFDM-OFDM coexisting systems with different subcarrier spacing. Matched filtering is used for signal detection.

Figure 7 presents the error performance of the coexisting signals in scenario-II, where independent OFDM and SEFDM signals are demodulated and detected separately using matched filtering and SD, respectively. Since signals of different formats are transmitted in the adjacent BWPs, SEFDM signals interfere with the OFDM signals and vice versa. The BER is noise-dominant at the high-noise range, whereas it turns to interference-dominant when the noise is relatively low. Thus, it can be seen that the BER performance of OFDM signals follows the ideal values at high-noise levels whilst slightly deviates when $E_{b} / N_{0}$ is larger than $3 \mathrm{~dB}$.

The BER performance for coexisting SEFDM signals with varying $\alpha$ in Scenario-III is illustrated in Fig. 8. Since both signal waveforms are non-orthogonal, under similar circumstances severe interference is shown in the BER degradation due to the high-level of bandwidth compression, especially when $E_{b} / N_{0}$ is greater than $5 \mathrm{~dB}$. Consequently, we consider employing LDPC coding algorithm to achieve error performance gain by performing interference cancellation in the next section.

\section{Improvement using $L D P C$}

Channel coding is commonly used in communication systems for the mitigation of channel impairments. Due to its

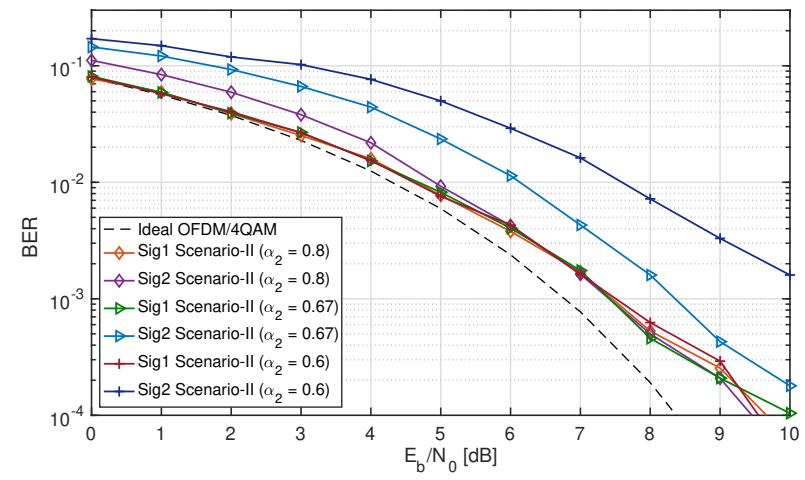

Fig. 7. Scenario-II: BER performance for OFDM-SEFDM coexistence with different subcarrier spacing due to varying values of $\alpha$ for SEFDM signal. $\mathrm{SD}$ is used for SEFDM signal detection. For OFDM signal detection matched filtering (also SD) is adopted.

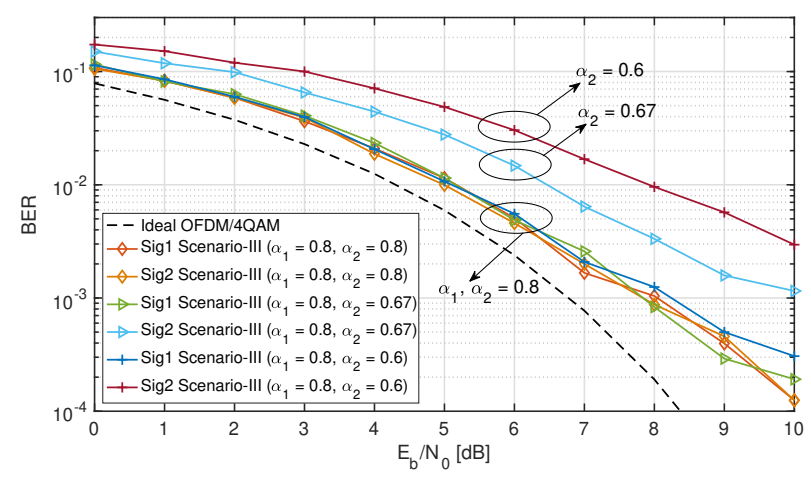

Fig. 8. Scenario-III: BER performance for SEFDM-SEFDM coexistence. Sphere decoder is used for signal detection.

high achievable data rate and low implementation complexity, LDPC [22] is used as standardised coding scheme for data channel for 5G NR [1]. Hence, LDPC coding is adopted for interference cancellation for the coexistence scenarios. We employ a fixed coding rate of $R_{c}=1 / 3$ for both SEFDM and OFDM signals and the 16-bit cyclic redundancy check (CRC) to assist BLER computation. Fig. 9 shows a general radio frame structure for both signals at $R_{c}=1 / 3$. Each frame (block) is defined to have 300 OFDM/SEFDM symbols, and each multicarrier symbol consists of 12 modulated 4QAM symbols. There are four puncturing strategies in 5G LDPC rate matching. For simplicity, this work follows the first punc-

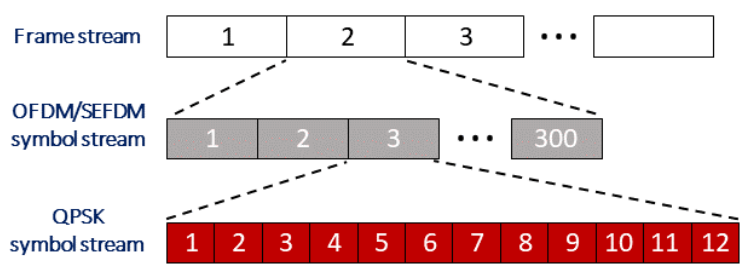

Fig. 9. Radio frame for coexistence scenarios. 


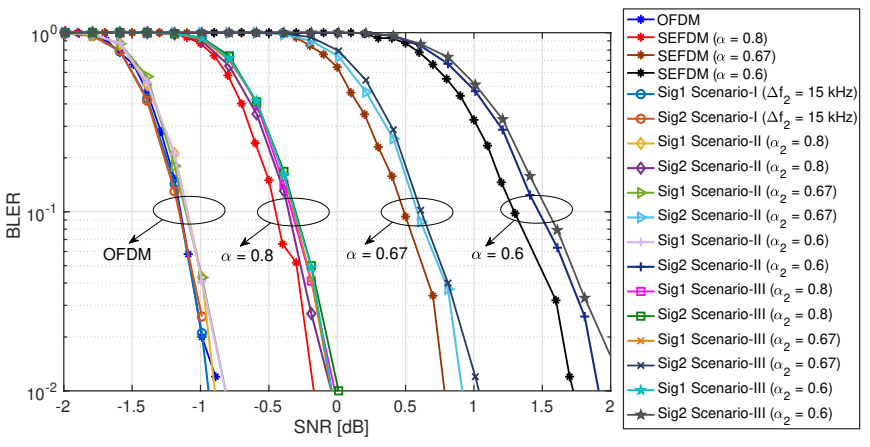

Fig. 10. BLER performance for the coexisting systems in three scenarios using LDPC coding, $\mathrm{N}=12$, coding rate $R_{c}=1 / 3$.

turing scheme, redundancy version RV0 [1]. For the channel decoding on the receiver side, the typical belief propagation decoding algorithm [22] is applied with 50 iterations.

To illustrate the practical advantages, we use SNR instead of theoretical $E_{b} / N_{0}$ for error performance assessment. Fig. 10 shows the BLER performance for the three scenarios of the different systems with varying configurations. For each system, the performance of two independent coexisting signals is evaluated separately. It is shown that all orthogonal signals achieve the same BLER performance whilst non-orthogonal signals have less than $0.3 \mathrm{~dB}$ variations when employing LDPC coding, regardless of the scenarios changes. The BLER categories associate with the four different values of $\alpha$, meaning an increasing bandwidth efficiency as $\alpha$ decreases at the cost of system performance. This leads to the conclusion that the negative effects of coexistence of orthogonal and non-orthogonal signals can be eliminated by adopting LDPC coding.

\section{CONCLUSION}

This paper presents the study of coexistence of orthogonal-OFDM and non-orthogonal-SEFDM multicarrier signals. Three typical coexistence scenarios are investigated, namely OFDM-OFDM, OFDM-SEFDM and SEFDM-SEFDM coexistence, where varying subcarrier spacings are considered under the flexible $5 \mathrm{G}$ NR numerology. This work reports the effects of the coexistence signalling using simulations, where both uncoded and LDPC coding assisted systems are studied. Results show that there is minor BER degradation for uncoded coexisting systems with non-orthogonal signals. The coexistence effects are ameliorated by LDPC coding and the BLER results demonstrate only negligible degradation for coexisting signals when compared to single signalling format system. This work offers insight for future heterogeneous system implementation considering non-orthogonal signalling coexistent with $5 \mathrm{G}$ NR.

\section{REFERENCES}

[1] 3GPP TS 38.212 version 15.2.0, "5G NR; multiplexing and channel coding," Rel. 15, Jul. 2018.

[2] E. Dahlman, S. Parkvall, and J. Sköld, $5 G$ NR: The Next Generation Wireless Access Technology. Academic Press, 2018.
[3] 3GPP TS 36.213 v.14.2.0, "LTE; evolved universal terrestrial radio access (E-UTRA); physical layer procedures," Rel. 14, Apr. 2017.

[4] B. Farhang-Boroujeny, "OFDM versus filter bank multicarrier," IEEE Signal Processing Magazine, vol. 28, no. 3, pp. 92-112, May 2011.

[5] N. Michailow, M. Matthe, I. Gaspar, A. Caldevilla, L. Mendes, A. Festag, and G. Fettweis, "Generalized frequency division multiplexing for 5th generation cellular networks," IEEE Transactions on Communications, vol. 62, no. 9, pp. 3045-3061, Sep. 2014.

[6] M. Kottkamp, R. . Schwarz, A. Pandey, D. Raddino, A. Rosessler, and R. Stuhlfauth, 5G New Radio: Fundamentals, Procedures, Testing Aspects. Rohde \& Schwarz GmbH \& Company KG, 2019. [Online]. Available: https://books.google.co.uk/books?id=wBOwwwEACAAJ

[7] I. Darwazeh, H. Ghannam, and T. Xu, "The first 15 years of sefdm: A brief survey," in 2018 11th International Symposium on Communication Systems, Networks \& Digital Signal Processing (CSNDSP). IEEE, 2018, pp. 1-7.

[8] J. B. Anderson, F. Rusek, and V. Öwall, "Faster-than-nyquist signaling," Proceedings of the IEEE, vol. 101, no. 8, pp. 1817-1830, 2013.

[9] M. Rodrigues and I. Darwazeh, "A spectrally efficient frequency division multiplexing based communications system," in Proc. 8th Int. OFDM Workshop, 2003, pp. 48-49.

[10] H. Ghannam, D. Nopchinda, M. Gavell, H. Zirath, and I. Darwazeh, "Experimental demonstration of spectrally efficient frequency division multiplexing transmissions at e-band," IEEE Transactions on Microwave Theory and Techniques, vol. 67, no. 5, pp. 1911-1923, 2019.

[11] T. Xu and I. Darwazeh, "Transmission experiment of bandwidth compressed carrier aggregation in a realistic fading channel," IEEE Transactions on Vehicular Technology, vol. 66, no. 5, pp. 4087-4097, 2016.

[12] _ "Non-orthogonal narrowband Internet of Things: A design for saving bandwidth and doubling the number of connected devices," IEEE Internet of Things Journal, vol. 5, no. 3, pp. 2120-2129, Jun. 2018.

[13] X. Liu and I. Darwazeh, "Quadrupling the data rate for narrowband internet of things without modulation upgrade," in 2019 IEEE 89th Vehicular Technology Conference (VTC Spring). IEEE, 2019, pp. 1-5.

[14] S. Isam and I. Darwazeh, "Design and performance assessment of fixed complexity spectrally efficient fdm receivers," in 2011 IEEE 73rd Vehicular Technology Conference (VTC Spring). IEEE, 2011, pp. 1-5.

[15] T. Xu, C. Masouros, and I. Darwazeh, "Waveform and space precoding for next generation downlink narrowband IoT," IEEE Internet of Things Journal, vol. 6, no. 3, pp. 5097-5107, Jun. 2019.

[16] I. Kanaras, A. Chorti, M. R. Rodrigues, and I. Darwazeh, "Spectrally efficient fdm signals: Bandwidth gain at the expense of receiver complexity," in 2009 IEEE International Conference on Communications. IEEE, 2009, pp. 1-6.

[17] X. Liu and I. Darwazeh, "Doubling the rate of spectrally efficient fdm systems using hilbert pulse pairs," in 2019 26th International Conference on Telecommunications (ICT). IEEE, 2019, pp. 192-196.

[18] S. Isam and I. Darwazeh, "Peak to average power ratio reduction in spectrally efficient fdm systems," in 2011 18th International Conference on Telecommunications. IEEE, 2011, pp. 363-368.

[19] H. Ghannam and I. Darwazeh, "Robust channel estimation methods for spectrally efficient fdm systems," in 2018 IEEE 87th Vehicular Technology Conference (VTC Spring). IEEE, 2018, pp. 1-6.

[20] S. Osaki, M. Nakao, T. Ishihara, and S. Sugiura, "Differentially modulated spectrally efficient frequency-division multiplexing," IEEE Signal Processing Letters, 2019.

[21] B. Yu, H. Zhang, X. Hong, C. Guo, A. P. T. Lau, C. Lu, and X. Dai, "Channel equalisation and data detection for sefdm over frequency selective fading channels," IET Communications, vol. 12, no. 18, pp. 2315-2323, 2018

[22] R. G. Gallager, Low-Density Parity-Check Codes. MIT Press, 1963. 